\title{
Nanoscale
}

Check for updates

Cite this: Nanoscale, 2021, 13, 16719

\section{Reduction in magnetic coercivity of Co nanomagnets by $\mathrm{Fe}$ alloying}

\author{
Hung-Hsiang Yang, ID *a,b Chuan-Che Hsu, ${ }^{c}$ Kanta Asakawa, ${ }^{a, d}$ Wen-Chin Lin ${ }^{c}$ and \\ Yukio Hasegawa id a
}

Received 27th July 2021, Accepted 10th September 2021 DOI: $10.1039 / \mathrm{d} 1 \mathrm{nr} 04862 \mathrm{~g}$ rsc.li/nanoscale

\begin{abstract}
We measured the magnetic hysteresis and coercivity of individual $\mathrm{Co}$ and $\mathrm{Co}_{0.8} \mathrm{Fe}_{0.2}$ bilayer nano-sized island structures formed on $\mathrm{Cu}$ (111) substrate using spin-polarized scanning tunneling microscopy. From the hysteresis taken on various sizes of islands, we found that the alloyed islands are ferromagnetic with out-of-plane magnetic anisotropy, same as the pure islands. Coercivity of the alloy islands, which is dependent on their size, was significantly reduced to $\approx 40 \%$ of that of the pure islands. Based on the Stoner-Wohlfarth model, we evaluated the amount of magnetic anisotropic energy and anisotropy constant for both pure and alloy islands. Since tunneling spectra taken on the alloy islands show upward shifts of the valence electronic states as compared to the pure ones, fewer electrons populated in the valence band of the alloy islands are presumably responsible for the reduction in the magnetic anisotropic energy.
\end{abstract}

\section{Introduction}

Nanomagnets have attracted much attention due to their high potential for applications. ${ }^{1,2}$ Owing to their reduced dimensions, the magnetic properties of nanomagnets are sensitive to both the type and size of the compositing materials. ${ }^{3}$ In order to control the properties of nanomagnets, it is, therefore, highly desirable to have a detailed understanding of the influences of compositing materials and their sizes on the magnetic properties of nanomagnets. Exploring the magnetism of nano-sized island structures requires magnetic measurements with nanometer-scale or atomic resolutions. Spin-polarized scanning tunneling microscopy (SP-STM) has been proved as the ideal instrument to investigate nano- and atomic-sized magnetic structures on surfaces. ${ }^{4-6}$ Several efforts have been made to resolve the magnetization reversal of elemental nanomagnets. ${ }^{3,7-11}$ In the case of thin films, on the other hand, the introduction of alloy composition is one of the most commonly applied techniques for manipulating their magnetic properties. In-depth understanding of alloyed thin films has been accumulated by extensive studies. ${ }^{12-16}$ However, studies on alloying effects in nanomagnets are still limited.

\footnotetext{
${ }^{a}$ Institute for Solid State Physics, the University of Tokyo, 5-1-5, Kashiwa-no-ha, KashiwaChiba 277-8581, Japan. E-mail: hung-hsiang.yang@kit.edu ${ }^{b}$ Physical Institute, Karlsruhe Institute of Technology, 76131 Karlsruhe, Germany ${ }^{c}$ Department of Physics, National Taiwan Normal University, Taipei, 116, Taiwan ${ }^{d}$ Department of Applied Physics, Tokyo University of Agriculture and Technology, Koganei, Tokyo 184-8588, Japan
}

Benefiting from the stable out-of-plane magnetization, nano-sized bilayer $\mathrm{Co}$ island structures on $\mathrm{Cu}$ (111) have been recognized as a standard sample to gauge the performance of SP-STM. ${ }^{17,18}$ Previous reports revealed that the coercivity of the Co islands depends on their sizes. ${ }^{3,10,11}$ A comprehensive experiment conducted by Ouazi et $a l^{3}{ }^{3}$ concluded that at an island size of around 7500 atoms $\left(\approx 210 \mathrm{~nm}^{2}\right.$ in area $)$, there is a crossover in the magnetization reversal mechanisms from an exchange-spring behavior to domain wall formation and propagation. Following the pure Co islands, $\mathrm{Co}_{1-x} \mathrm{Fe}_{x}$ alloy islands were investigated by Yang et al. ${ }^{19}$ When alloying Co with $\mathrm{Fe}$, the minority $\mathrm{d}$ electronic state shifts its energy upward, which indicates a reduction in the total number of $\mathrm{d}$ electrons of the nano-islands. For the islands with $x=0,0.2$, and 0.5 , the out-of-plane magnetic anisotropy is found preserved.

Different from the case of bulk, the magnetic anisotropic energy (MAE) in thin films is dominated by the surface/interface atoms. ${ }^{20}$ In the case of bilayer Co islands, the MAE is purely contributed by the surface/interface atoms. The MAE of the bilayer Co islands can be acquired by measuring the coercivity as a function of island size $\mathrm{e}^{3}$ and alloying with Fe provides opportunities to modify it. ${ }^{19}$

In this study, we employed SP-STM and spectroscopy to investigate the magnetization reversal of individual Co and $\mathrm{Co}_{0.8} \mathrm{Fe}_{0.2}$ islands on $\mathrm{Cu}$ (111). The coercivity of the islands was extracted from the magnetic hysteresis of spin-dependent tunneling conductance, which was measured during a sweep of a perpendicularly applied external magnetic field. By analyzing the results using the Stoner-Wohlfarth model, we found a 
reduction in MAE of individual alloy nanomagnets compared with that of pure ones of the same size.

\section{Methods}

The Co/Cu (111) sample was prepared by evaporating Co on $\mathrm{Cu}$ (111) surface that had been cleaned through repetitive cycles of $\mathrm{Ar}^{+}$sputtering and annealing $(\approx 1000 \mathrm{~K}$ under ultra-high vacuum (UHV) with a base pressure of $5 \times 10^{-8} \mathrm{~Pa}$ ). The $\mathrm{Co}_{0.8} \mathrm{Fe}_{0.2} / \mathrm{Cu}(111)$ sample was prepared by co-depositing $\mathrm{Co}$ and $\mathrm{Fe}$ on $\mathrm{Cu}$ (111) surface. The ratio between Co and Fe was controlled by regulating the deposition rate of Co and Fe. The deposition rate of $\mathrm{Co}(\mathrm{Fe})$ was set at $3.63 \pm 0.03(3.64 \pm 0.02)$ $\mathrm{ML} \min ^{-1}$ and the $\mathrm{Cu}$ (111) substrate was maintained at room temperature during the deposition. STM measurements were performed with a low-temperature (6 K) UHV STM (Unisoku USM-1300S with an RHK R9 controller), which was equipped with superconducting magnets for the application of magnetic fields perpendicular $\left(\left|B_{\text {ext }}\right| \leq 2 \mathrm{~T}\right)$ to the sample surface. The spin-polarized tip was prepared by depositing Fe on an electrochemically etched $\mathrm{W}$ tip that had been pre-annealed in UHV at $\approx 2000 \mathrm{~K}$. The tunneling spectra and images were recorded in a standard lock-in method with the peak-to-peak bias voltage modulation $\left(V_{\mathrm{mod}}^{\mathrm{PP}}\right.$ ) of 10 or $50 \mathrm{mV}$ at $971 \mathrm{~Hz}$. All images were processed using Nanotech WSxM. ${ }^{21}$ Magnetic hysteresis loops were acquired by measuring the differential conductance $(\mathrm{d} I)$ $\mathrm{d} V$ ) set at majority/minority states as a function of the external magnetic field at a designated location on individual Co and $\mathrm{Co}_{0.8} \mathrm{Fe}_{0.2}$ islands.

\section{Results and discussion}

Deposition of Co with 0.6 monolayer coverage on the $\mathrm{Cu}$ (111) substrate forms triangular-shaped bilayer Co islands on the surface (Fig. 1a). Depending on the stacking crystallographic orientation with respect to $\mathrm{Cu}$ (111), two types of Co islands were observed: unfaulted and faulted islands. The former follows the same stacking as $\mathrm{Cu}$ (111) and for the latter the $\langle 112\rangle$ orientation is rotated by $180^{\circ} \cdot{ }^{19,22,23}$ From the peak energy of electronic states, the upward- and downward-pointed islands in the STM images are identified as faulted and unfaulted islands, respectively. ${ }^{19,22}$ The $\mathrm{Co}_{0.8} \mathrm{Fe}_{0.2}$ islands formed on $\mathrm{Cu}$ (111) show the morphology similar to that of pure Co islands (Fig. 1b). The $\mathrm{Co}_{0.8} \mathrm{Fe}_{0.2}$ islands also exhibit the faulted and unfaulted stackings. The corresponding differential tunneling conductance $(\mathrm{d} I / \mathrm{d} V)$ maps of Co and $\mathrm{Co}_{0.8} \mathrm{Fe}_{0.2}$ islands are presented in the right panel of Fig. 1a and $b$, respectively. Within an individual island, the Co islands show homogeneous $\mathrm{d} I / \mathrm{d} V$ intensity at the sample voltage of $-0.3 \mathrm{~V}$, which is at the vicinity of the minority Co $\mathrm{d}_{3 z^{2}-r^{2}}$ state. ${ }^{24}$ Due to the incorporation of Fe atoms, the $\mathrm{d} I / \mathrm{d} V$ intensity varies within the $\mathrm{Co}_{0.8} \mathrm{Fe}_{0.2}$ islands. ${ }^{19}$

Spin-polarized measurements are carried out using an Fecoated $\mathrm{W}$ tip, which exhibits superparamagnetic response. ${ }^{25}$ The out-of-plane magnetization component of the tip is controlled by the external out-of-plane magnetic field $\left(B_{\text {ext }}\right)$. The schematics presented in the upper panel of Fig. 1c illustrate the magnetization direction of the tip with respect to the sample surface. Under the application of the external magnetic field, the tip magnetization points towards the out-of-plane

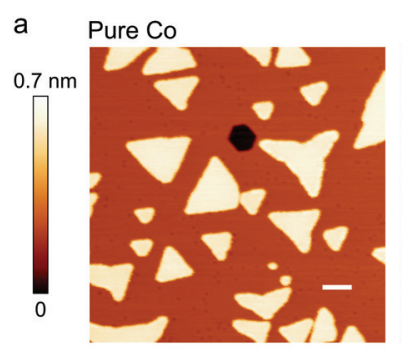

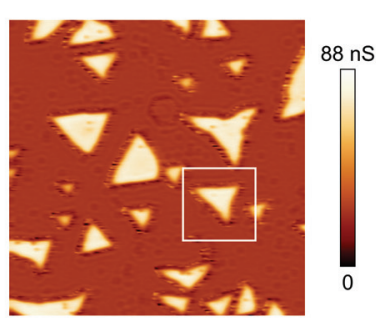

c

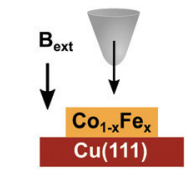

$-0.3 \mathrm{~T}$

$-0.3 V$

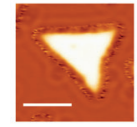

d
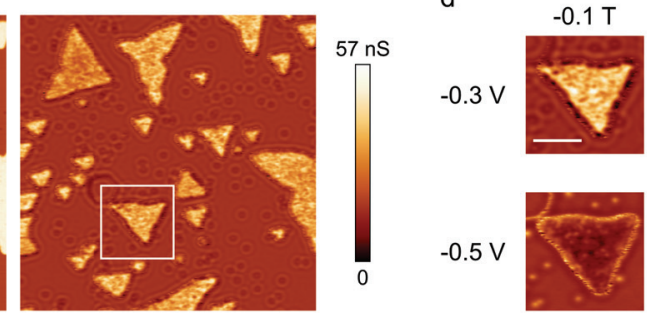
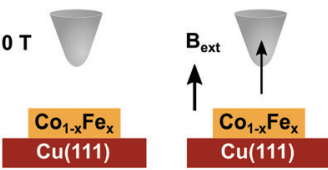

$0 \mathrm{~T}$

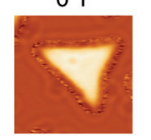

$0.3 \mathrm{~T}$
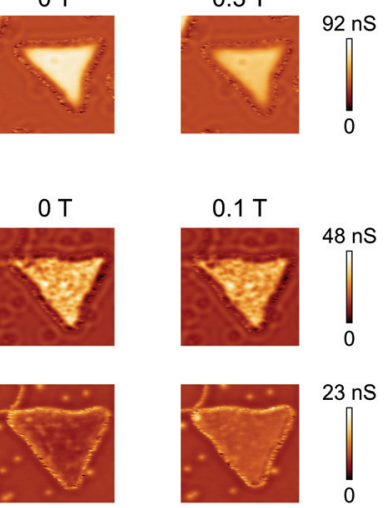

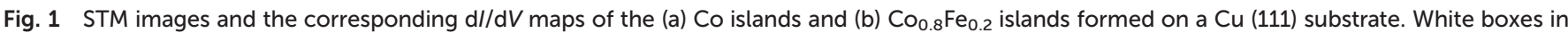

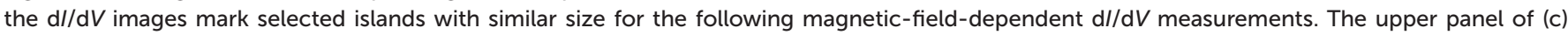

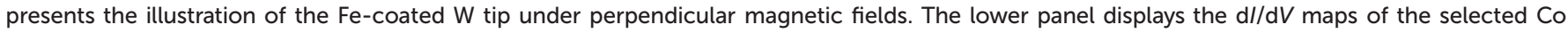

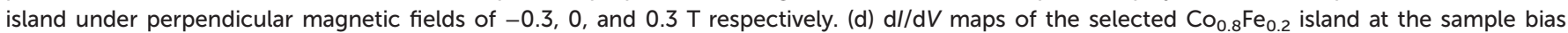

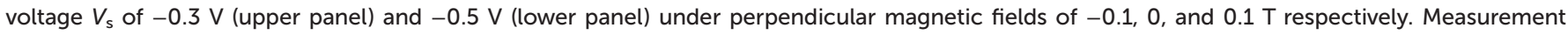

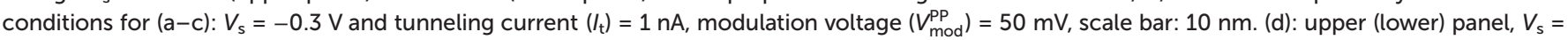
$-0.3 \mathrm{~V}(-0.5 \mathrm{~V}), I_{\mathrm{t}}=1 \mathrm{nA}, V_{\mathrm{mod}}^{\mathrm{PP}}=50 \mathrm{mV}$, scale bar: $10 \mathrm{~nm}$. 
direction. The lower panel of Fig. 1c shows the $\mathrm{d} I / \mathrm{d} V$ maps of a Co island under magnetic fields $-0.3,0$, and $0.3 \mathrm{~T}$ respectively. The sample bias voltage $V_{\mathrm{s}}$ was set at $-0.3 \mathrm{~V}$, which corresponds to the minority state, and prior to the measurement the magnetization of the Co islands was saturated by applying an out-of-plane magnetic field of $2 \mathrm{~T}$. The $\mathrm{d} I / \mathrm{d} V$ maps reveal pronounced intensity variation of the Co islands depending on the magnetic fields. At the sample bias of $-0.3 \mathrm{~V}$, the $\mathrm{d} I / \mathrm{d} V$ intensity of the Co island is the highest at $-0.3 \mathrm{~T}$, intermediate at $0 \mathrm{~T}$, and the lowest at $0.3 \mathrm{~T}$. Since the Co islands are perpendicularly magnetized single-domain nanomagnets, the observed contrast is understood as the superparamagnetic response of the out-of-plane-component of the tip magnetization; the out-of-plane component of the tip magnetization is antiparallel at $-0.3 \mathrm{~T}$, zero at $0 \mathrm{~T}$, and parallel at $0.3 \mathrm{~T}$ to that of the Co island. Similar experiments were performed on $\mathrm{Co}_{0.8} \mathrm{Fe}_{0.2}$ with a smaller range of magnetic fields $(-0.1,0$, and $0.1 \mathrm{~T}$ ). Fig. 1d displays the $\mathrm{d} I / \mathrm{d} V$ images of an individual $\mathrm{Co}_{0.8} \mathrm{Fe}_{0.2}$ island at sample voltages of $-0.3 \mathrm{~V}$ (minority state, upper panel) and $-0.5 \mathrm{~V}$ (majority state, lower panel). The $\mathrm{d} I /$ $\mathrm{d} V$ intensity taken at the minority (majority) state decreases (increases) with the increment of the magnetic field, consistent with the superparamagnetic response of the tip magnetization.

The spin-polarized states of the Co and $\mathrm{Co}_{0.8} \mathrm{Fe}_{0.2}$ islands were investigated using spin-polarized tunneling spectra. Fig. 2a displays the field-dependent spin-polarized $\mathrm{d} I / \mathrm{d} V$ spectra taken on an unfaulted Co island. The measured position is marked in the STM image in Fig. 2a. Prior to the measurements, the magnetizations of the sample were saturated by applying an out-of-plane magnetic field of $2 \mathrm{~T}$.

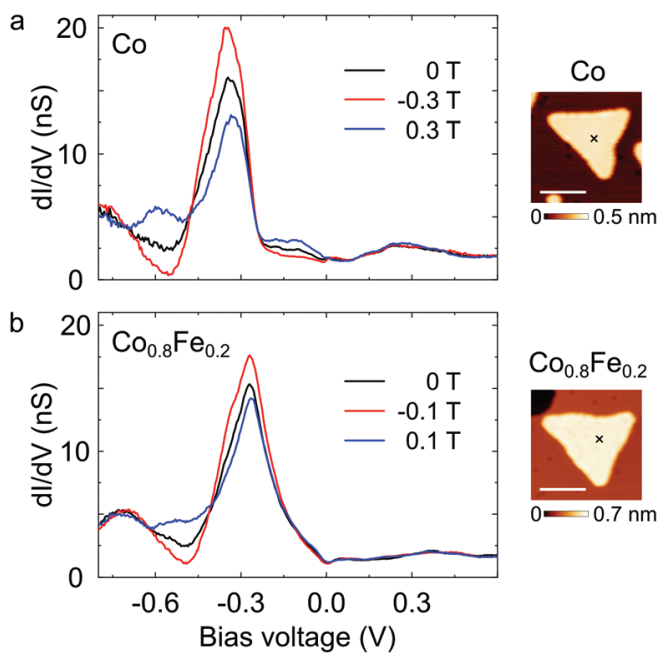

Fig. 2 (a) Spin-polarized $d / / d V$ spectra measured on an unfaulted Co island at $0 \mathrm{~T}$ (black), $-0.3 \mathrm{~T}$ (red), and $0.3 \mathrm{~T}$ (blue). The measured position is marked by a cross in the inset STM image. (b) Spin-polarized $\mathrm{d} / / \mathrm{d} V$ spectra measured on an unfaulted $\mathrm{Co}_{0.8} \mathrm{Fe}_{0.2}$ island at $0 \mathrm{~T}$ (black), $-0.1 \mathrm{~T}$ (red), and $0.1 \mathrm{~T}$ (blue). The measured position is marked by a cross in the inset STM image. STM images: $V_{\mathrm{s}}=-300 \mathrm{mV}, I_{\mathrm{t}}=1 \mathrm{nA}$, scale bar: $10 \mathrm{~nm}$. $\mathrm{d} l / \mathrm{d} V$ spectra: $V_{\mathrm{s}}=600 \mathrm{mV}, I_{\mathrm{t}}=1 \mathrm{nA}, V_{\text {mod }}^{\mathrm{PP}}=10 \mathrm{mV}$.
Accordingly, the magnetization relation between the out-ofplane component of the tip and the Co island corresponds to antiparallel at $-0.3 \mathrm{~T}$ (red) and parallel at $0.3 \mathrm{~T}$ (blue). At $0 \mathrm{~T}$, the net moment of the tip is zero, and therefore the spectrum is spin-averaged. Pronounced differences in the $\mathrm{d} I / \mathrm{d} V$ intensity appear at the $V_{\mathrm{s}}$ of $-0.33 \mathrm{~V}$ and $-0.55 \mathrm{~V}$, which corresponds to the minority and majority states, respectively. ${ }^{4,5,24}$ Similar measurements with smaller magnetic fields were performed on an unfaulted $\mathrm{Co}_{0.8} \mathrm{Fe}_{0.2}$ island. The $\mathrm{d} I / \mathrm{d} V$ spectra at $-0.1,0$, and $0.1 \mathrm{~T}$ are presented in Fig. $2 \mathrm{~b}$ as red, black, and blue curves, respectively. The measured position is marked in the STM image on the right panel of Fig. $2 \mathrm{~b}$. The $\mathrm{d} I / \mathrm{d} V$ intensity contrasts at $V_{\mathrm{s}}$ of $-0.28 \mathrm{~V}$ and $-0.5 \mathrm{~V}$. Comparing with the case of the Co island, the spin-polarized states of the alloy island shift towards the Fermi level by around $0.05 \mathrm{~V}$. The shifts of the state energy result from the hole-doping by the incorporation of Fe atoms. ${ }^{19}$

Magnetic hysteresis loops of Co islands with different sizes are presented in Fig. 3a-c. Each loop was measured at the position marked by the cross in the respective STM image with $V_{\mathrm{s}}=$ $-0.3 \mathrm{~V}$ to probe the minority state. The hysteresis loop exhibits a butterfly-like feature that is composed of a gradual increase and an abrupt drop during the magnetic sweep. The applied magnetic field at which the abrupt drop occurs depends on Co islands whereas the gradual increase is the same regardless of the islands. The gradual increase is thus due to the variation in the tip magnetization whereas the abrupt drop is due to a switch of the magnetization of the Co island. The switching fields $\left(B_{\mathrm{sw}}\right)$ are therefore extracted from the magnetic field at which abrupt drops occur in the butterfly-shaped hysteresis loops. For a Co island that contains around 1600 atoms, $B_{\mathrm{sw}}$ is $0.14 \mathrm{~T}$ (Fig. 3a). The islands (Fig. 3b and c) that are composed of around 3200 and 4500 atoms exhibit the switching fields of 0.24 and $0.32 \mathrm{~T}$, respectively. Similar measurements were performed on $\mathrm{Co}_{0.8} \mathrm{Fe}_{0.2}$ islands with $V_{\mathrm{s}}=-0.5 \mathrm{~V}$ (Fig. 3d-f), which probed majority states and thus made the loops inverted. The hysteresis loop of $\mathrm{Co}_{0.8} \mathrm{Fe}_{0.2}$ islands that included around 1600, 3200, and 4500 atoms exhibited the switching fields of $0.05,0.08$, and $0.11 \mathrm{~T}$, respectively. The $\mathrm{Co}_{0.8} \mathrm{Fe}_{0.2}$ islands exhibit significantly smaller switching fields than the pure Co islands.

The switching fields of pure Co islands are found much smaller compared with those of a previous study by Ouazi et $a .^{3}$ The main reason was that the magnetic field strayed from the Fe-coated tip. ${ }^{26}$ One of the characteristics of the tipinduced stray fields is their locality. In order to demonstrate that, we performed the following experiment. First, we scanned a large area $\left(200 \times 200 \mathrm{~nm}^{2}\right)$ with the spin-polarized tip under the external magnetic field of $2 \mathrm{~T}$ in order to saturate the magnetization of all the islands. Fig. 4 a displays the simultaneously taken $\mathrm{d} I / \mathrm{d} V$ map with $V_{\mathrm{s}}=-0.3 \mathrm{~V}$ to confirm the saturation; all the islands show a uniformly low $\mathrm{d} I / \mathrm{d} V$ value, implying similar magnetization direction. Next, we set the magnetic field in the opposite polarity $(-0.1 \mathrm{~T})$. Under the field, the magnetizations of the tip and the islands are expected in the antiparallel configuration. Fig. 4b presents a 
a
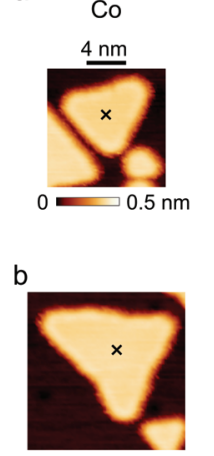

C

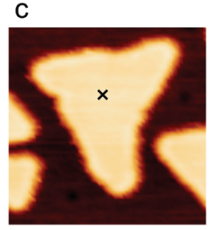

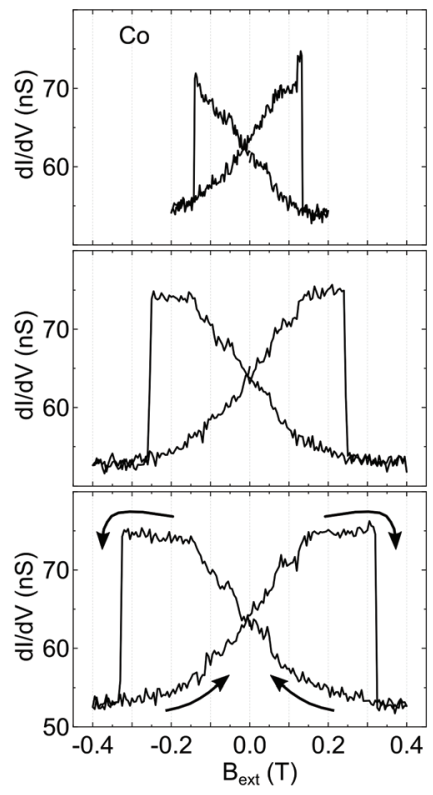

d

$\mathrm{Co}_{0.8} \mathrm{Fe}_{0}$

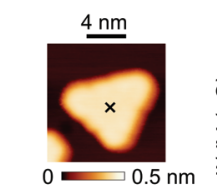

e
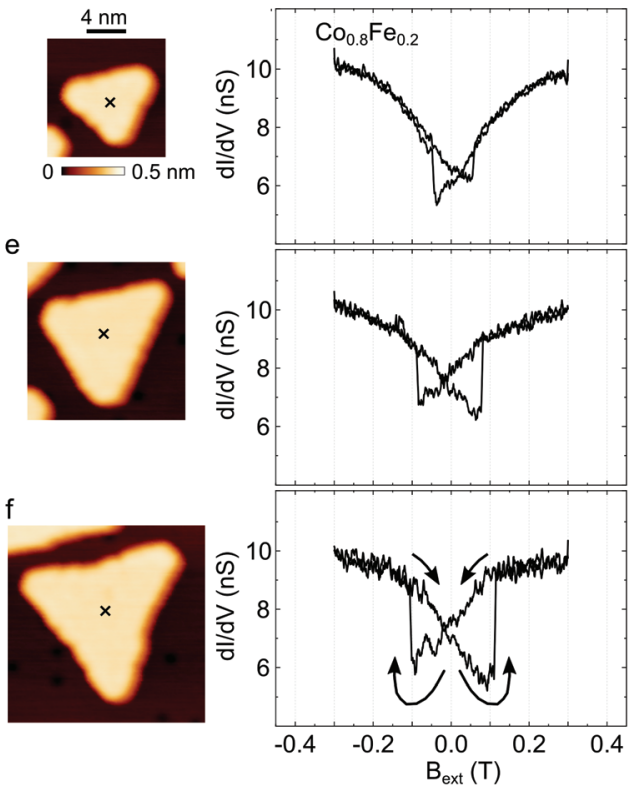

Fig. 3 (a-c) STM images of unfaulted Co islands with the corresponding magnetic hysteresis loops taken at the marked sites respectively. (d-f) STM images of unfaulted $\mathrm{Co}_{0.8} \mathrm{Fe}_{0.2}$ islands with the corresponding magnetic hysteresis loops taken at the marked sites respectively. Note that all the STM images are in the same scale. STM images $(\mathrm{a}-\mathrm{c}) V_{\mathrm{s}}=-300 \mathrm{mV}, l_{\mathrm{t}}=1 \mathrm{nA}$, (d-f) $V_{\mathrm{s}}=-500 \mathrm{mV}, l_{\mathrm{t}}=1 \mathrm{nA}$, scale bar: $4 \mathrm{~nm}$. Magnetic hysteresis loops $(\mathrm{a}-\mathrm{c}) V_{\mathrm{s}}=-300 \mathrm{mV}, I_{\mathrm{t}}=1 \mathrm{nA}, V_{\text {mod }}^{\mathrm{PP}}=50 \mathrm{mV}$, (d-f) $V_{\mathrm{s}}=-500 \mathrm{mV}, I_{\mathrm{t}}=1 \mathrm{nA}, V_{\text {mod }}^{\mathrm{PP}}=50 \mathrm{mV}$. The arrows in the hysteresis loops indicate the sweeping directions.

$\mathrm{d} I / \mathrm{d} V$ map taken under the field. Most of the islands exhibit larger $\mathrm{d} I / \mathrm{d} V$ intensity except the small islands. The larger $\mathrm{d} I /$ $\mathrm{d} V$ intensity indicates the antiparallel magnetization configuration with the tip. The smaller $\mathrm{d} I / \mathrm{d} V$ intensity found on the smaller islands indicates the islands undergo a magnetic reversal and thus their magnetizations are aligned with the tip.

Then we scanned a zoomed area $\left(60 \times 60 \mathrm{~nm}^{2}\right)$ marked by the square in Fig. $4 \mathrm{~b}$ under various magnetic fields of -0.1 , $-0.2,-0.3$, and $-0.4 \mathrm{~T}$. The corresponding $\mathrm{d} I / \mathrm{d} V$ maps are presented in Fig. 4c-f. At $-0.1 \mathrm{~T}$ (Fig. 4c), most islands remain antiparallel to the tip, and only two small islands at the bottom of the image are switched. At $-0.2 \mathrm{~T}$ (Fig. 4d), more islands are switched and a switching is observed even during the scanning (marked by arrows). More islands are switched and more switching events are observed at $-0.3 \mathrm{~T}$ (Fig. 4e). Eventually at $-0.4 \mathrm{~T}$ (Fig. 4f), all the islands are switched and align with the tip magnetization. Finally, we went back to the original scan size and took the $\mathrm{d} I / \mathrm{d} V$ map, which is presented in Fig. 4g. In the map, only the islands inside and in the vicinity ( $\approx 20 \mathrm{~nm}$ from the edge) of the zoomed area are switched, demonstrating the confined stray field from the scanned area and thus from the tip apex.

As mentioned above, the magnetic hysteresis loops are composed of two parts: gradual increase and abrupt drop in the case of the minority-state probing (e.g. Fig. 2a-c). The gradual part is due to the superparamagnetic-like behavior of the tip magnetization and the abrupt part is due to the switching of the hard ferromagnetic Co islands. In order to characterize the magnetic response of the tip, we extracted gradual parts from a butterfly-like hysteresis loop taken on the island investigated in Fig. 2a and displayed in Fig. 5a. The black (red) data show the ones taken during the upward (downward) sweep from $-0.3 \mathrm{~T}(0.3 \mathrm{~T})$ to $0.3 \mathrm{~T}(-0.3 \mathrm{~T})$. In order to compensate the switching of the Co island, data taken during the downward sweep were inverted with respect to a horizontal line passing through the cross point at the zero field. The data can be fitted nicely with the Langevin function as depicted with a blue line. From the fitting we safely conclude that the tip magnetization behaves as a superparamagnet and the magnetic moment is given as $138 \mu_{\mathrm{B}}$, which agrees with the typical values $\left(100-2000 \mu_{\mathrm{B}}\right)$ of Fe-coated $\mathrm{W}$ tips. ${ }^{25}$

In order to estimate the total magnetic field acting on the islands, the stray field from the tip needs to be considered. The amount of the stray field is assumed proportional to the tip magnetization, $M\left(B_{\text {ext }}\right)$, the blue curve in Fig. $5 \mathrm{a}$, which varies as a function of the external field $B_{\text {ext }}$. The total magnetic field is thus expressed as

$$
B_{\text {total }}=a M\left(B_{\text {ext }}\right)+B_{\text {ext }}
$$

The coefficient $a$ can be obtained by substituting the reference data from ref. 3 into the following equation:

$$
B_{\mathrm{sw}}^{\mathrm{ref}}=a M\left(B_{\mathrm{SW}}\right)+B_{\mathrm{Sw}}
$$

where $B_{\mathrm{sw}}^{\mathrm{ref}}$ is the switching field from the reference and $B_{\mathrm{sw}}$ corresponds to the result of this study with a similar-sized Co 
a

$2.0 \mathrm{~T}$

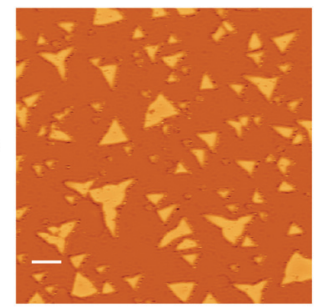

b

$-0.1 \mathrm{~T}$

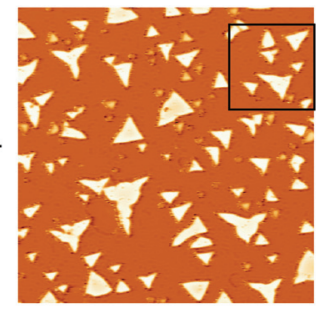

g

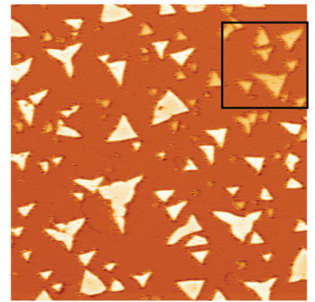

0

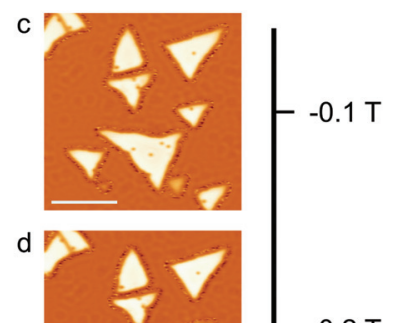

$-0.2 \mathrm{~T}$

e

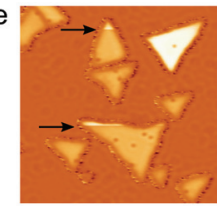

f

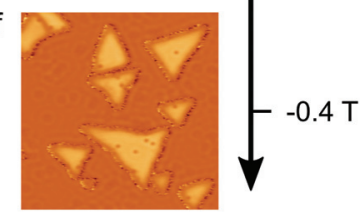

Fig. 4 (a) $d / / d V$ map at $2.0 \mathrm{~T}\left(200 \times 200 \mathrm{~nm}^{2}\right)$. (b) $\mathrm{d} / / \mathrm{d} V$ map at $-0.1 \mathrm{~T}$, the black square marks the zoomed scan area $\left(60 \times 60 \mathrm{~nm}^{2}\right)$ for $(\mathrm{c}-\mathrm{f})$. $\mathrm{d} / / \mathrm{d} V$ maps of the zoomed area taken under $-0.1 \mathrm{~T}$ (c), $-0.2 \mathrm{~T}(\mathrm{~d}),-0.3 \mathrm{~T}$ (e), and $-0.4 \mathrm{~T}(\mathrm{f})$. The arrows mark switching of the magnetization that occurred during scanning. (g) The zoomed-out $d / / d V$ map after $(c-f)$. Measurement conditions: $V_{\mathrm{s}}=-0.3 \mathrm{~V}$ and $I_{\mathrm{t}}=1 \mathrm{nA}, V_{\text {mod }}^{\mathrm{PP}}=50 \mathrm{mV}$, scale bar: $20 \mathrm{~nm}$.

island. The inset of Fig. $5 \mathrm{~b}$ shows the coefficients that were acquired from the data of different sized islands. The coefficient $a$ remains more or less constant regardless of the size and gives an average value of 1.69, which is then used for calculating the total magnetic field. The calibrated total magnetic field acting on the islands are plotted in Fig. $5 \mathrm{~b}$.

The coercivity of each island was extracted with the calibrated magnetic field and summarized in Fig. 6 . The square (circular) data points represent the coercivity $B_{\mathrm{c}}$ of the Co $\left(\mathrm{Co}_{0.8} \mathrm{Fe}_{0.2}\right)$ islands. The filled (hollow) data points represent the coercive fields extracted from the downward (upward) sweeps. The discrepancy between up- and downward sweeps is presumably due to the stray field from nearby islands, which may change during the sweep. ${ }^{3,4}$ As concluded by Ouazi et al., ${ }^{3}$ the mechanism of the Co island magnetization reversal shows a crossover from an exchange-spring behavior to domain wall formations at the island size of around 7500 atoms $\left(210 \mathrm{~nm}^{2}\right)$. The emergence of domain wall formation at larger islands gives rise to the observed nonmonotonic dependence of $B_{\mathrm{c}}$ on the number of atoms $N$. Analogous with Co islands, the magnetization reversal of $\mathrm{Co}_{0.8} \mathrm{Fe}_{0.2}$ islands shows the similar crossover at an island size around 7500 atoms. The main difference lies on the reduced coercivity of the $\mathrm{Co}_{0.8} \mathrm{Fe}_{0.2}$ islands.

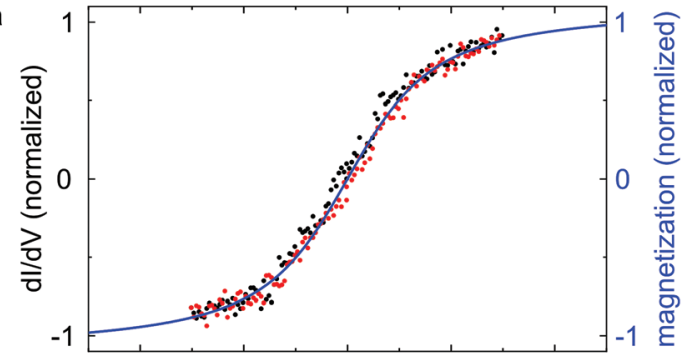

b

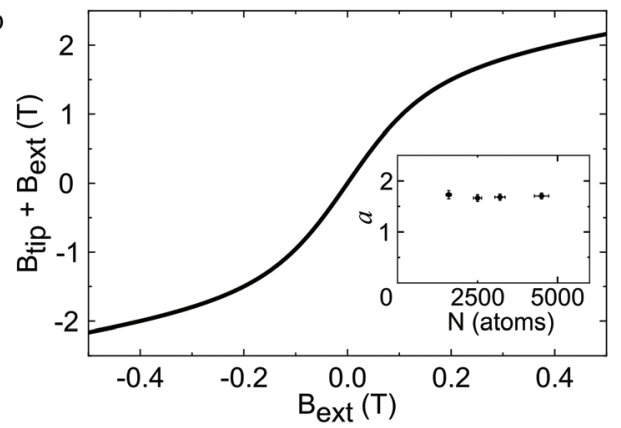

Fig. 5 (a) $d / / d V$ spectra as a function of the external magnetic fields during the upward (black) and downward (red) sweeps taken on the island shown in Fig. 2a. Data taken during the downward sweep were inverted with respect to a horizontal line passing through the cross point at the zero field. The out-of-plane component of the magnetization is fitted with a Langevin function as plotted with the blue curve. Measuring parameters: $V_{\mathrm{s}}=-300 \mathrm{mV}, I_{\mathrm{t}}=1 \mathrm{nA}, V_{\text {mod }}^{\mathrm{PP}}=50 \mathrm{mV}$. (b) Calibrated total magnetic field acting on the islands as a function of the applied external magnetic field. The inset shows the coefficient $a$ as a function of the island size.

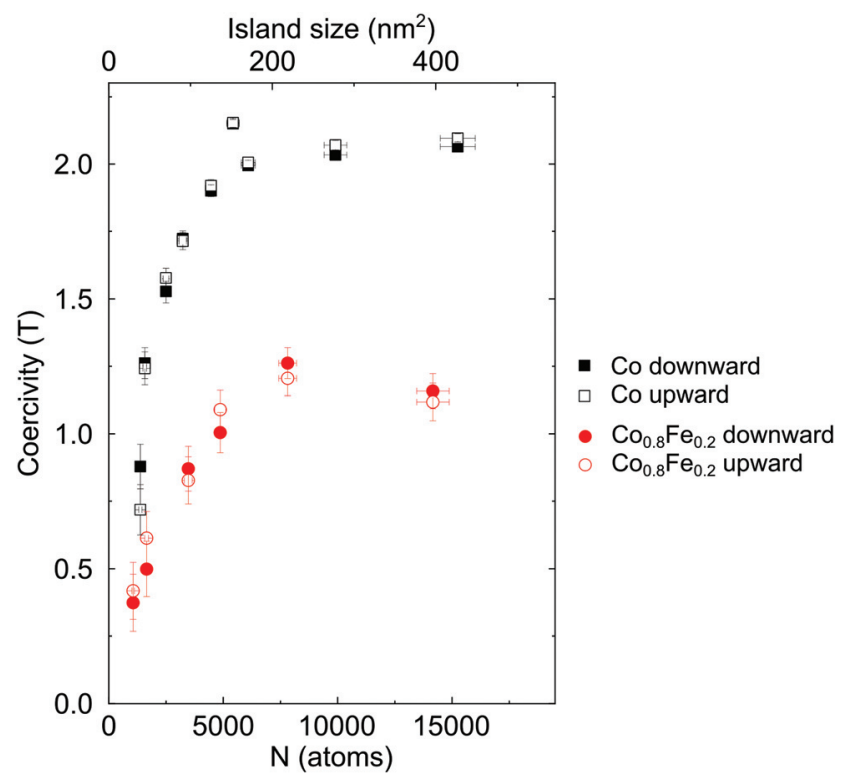

Fig. 6 Coercivity of individual Co (black squares) and $\mathrm{Co}_{0.8} \mathrm{Fe}_{0.2}$ (red circles) islands of different sizes extracted from the upward (solid) and downward (hollow) sweeps of the magnetic hysteresis loops. Magnetization reversal due to domain wall formation emerges when the island size exceeds around 7500 atoms ${ }^{3}$ for both $\mathrm{Co}$ and $\mathrm{Co}_{0.8} \mathrm{Fe}_{0.2}$ islands. 
For a single-domain system, the coercivity strongly correlates with the magnetic anisotropy. ${ }^{27}$ In the following, we discuss such a correlation for the islands in the single-domain regime ( $<8000$ atoms). Considering a system under a magnetic field $B$ in the opposite direction with respect to its magnetization, the apparent barrier $E^{27}$ for reversing the magnetization is expressed as

$$
E(B)=\Delta E\left(1-N \frac{\mu B_{\mathrm{c}}}{2 \Delta E}\right)^{2}
$$

where $\mu$ corresponds to the magnetic moment per atom and $\Delta E$ is the energy difference between the out-of-plane and inplane magnetizations, that is, MAE. When the external field reaches the coercive field, $B=B_{\mathrm{c}}$, the thermal energy at temperature $T$ overcomes the barrier. As a result, thermally activated magnetization reversal occurs. ${ }^{28}$ Eqn (3) is thus expressed as

$$
k_{\mathrm{B}} T \ln \frac{t}{\tau_{0}}=\Delta E\left(1-N \frac{\mu B_{\mathrm{c}}}{2 \Delta E}\right)^{2}
$$

where $\tau_{0}$ is the exponential prefactor for the switching, which is set to $10^{-10} \mathrm{~s}^{29}$ The measuring time $t$ is around $1 \mathrm{~s}$. The energy barriers $\Delta E$ of Co (black) and $\mathrm{Co}_{0.8} \mathrm{Fe}_{0.2}$ (red) as a function of $N$ are extracted using eqn (4) and plotted in Fig. 7. The dependence of $\Delta E$ on $N$ is fitted with a linear function that is presented in black and red lines for Co and $\mathrm{Co}_{0.8} \mathrm{Fe}_{0.2}$ islands, respectively. According to the Stoner-Wohlfarth model for a single-domain ferromagnet with uniaxial anisotropy, MAE is described as

$$
\Delta E=K N
$$

where $K$ is the anisotropy constant of the sample. Therefore, the slopes fitted from the data in Fig. 7 manifest $K_{\mathrm{Co}}=0.139 \pm$ $0.006 \mathrm{meV}$ per atom for $\mathrm{Co}$ islands and $K_{\mathrm{CoFe}}=0.082 \pm$ $0.005 \mathrm{meV}$ per atom for $\mathrm{Co}_{0.8} \mathrm{Fe}_{0.2}$ islands. The value of $K_{\mathrm{Co}}$

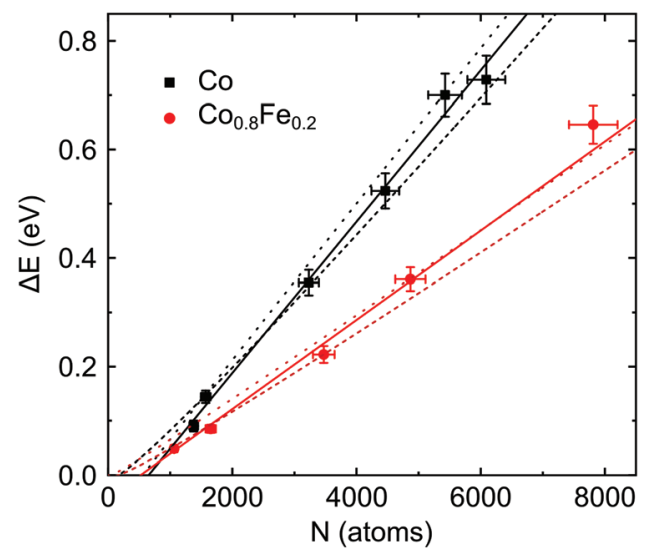

Fig. 7 Energy differences between the out-of-plane and in-plane magnetizations for $\mathrm{Co}$ (black) and $\mathrm{Co}_{0.8} \mathrm{Fe}_{0.2}$ (red) islands extracted using eqn (3) and (4). The slopes of the linear fits (solid lines) give the anisotropy constant of $\mathrm{Co}$ and $\mathrm{Co}_{0.8} \mathrm{Fe}_{0.2}$, islands, respectively. The dotted and dashed lines are the plots of the function $K\left(N-N_{\text {rim }}\right)$ for the rim widths of 1 and 2 atomic rows, respectively. agrees with a previous study. ${ }^{3}$ The smaller value of $K_{\mathrm{CoFe}}$ corresponds to the observed reduction in the coercivity of the $\mathrm{Co}_{0.8} \mathrm{Fe}_{0.2}$ islands. The extrapolation of the fitted lines give offsets in the number of atoms $\left(N_{0}\right)$ of $650 \mathrm{pm} 180$ and $520 \pm$ 140 atoms for $\mathrm{Co}$ and $\mathrm{Co}_{0.8} \mathrm{Fe}_{0.2}$ islands, respectively. These offsets indicate that the actual number of atoms that contribute to the anisotropy is reduced to $N-N_{0}$. The origin of the offset is attributed to the influence of the rim atoms of the islands ${ }^{3}$ the rim atoms have magnetic properties different from the other island-forming atoms and thus do not contribute to the magnetic anisotropy. Here we consider the rim of 1 and 2 atomic rows for both the $\mathrm{Co}$ and $\mathrm{Co}_{0.8} \mathrm{Fe}_{0.2}$ islands. Using the fitted values of $K_{\mathrm{Co}}$ and $K_{\mathrm{CoFe}}$, the functions $K(N-$ $N_{\text {rim }}$ ) for 1 and 2 atomic-wide rims are plotted in Fig. 7 with dotted and dashed lines, respectively. The experimental data land on the vicinities of the lines, which implies that the effective rim irrelevant to the magnetic anisotropy is 1 or 2 atoms wide. After incorporating $\mathrm{Fe}$ atoms, the anisotropy constant of the Co nano-islands is reduced by $0.057 \pm 0.008 \mathrm{meV}$ per atom. One of the origins of the anisotropy reduction arises from the changes in the number of valence electrons. Referring to the calculation results by Daalderop et al. ${ }^{30}$ the anisotropic energy of Co exhibits a reduction of $\approx 0.05 \mathrm{meV}$ per atom when the number of the valence electrons decreases from 9 to 8.9. Such an anisotropy reduction coincides with the observed difference between $K_{\mathrm{Co}}$ and $K_{\mathrm{CoFe}}$ in this study. The decrease in valence electrons is also expected when alloying Co with Fe since pure Fe contains one electron less than the pure Co. Furthermore, in the spin-polarized tunneling spectra (Fig. 2), both the majority and the minority states of the $\mathrm{Co}_{0.8} \mathrm{Fe}_{0.2}$ island shift upward in energy as compared to the Co island, indicating reduced electron population in the valence band of the alloy island. This qualitative analysis suggests that the reduced anisotropy of $\mathrm{Co}_{0.8} \mathrm{Fe}_{0.2}$ islands comes from the reduced number of valence electrons as compared to Co islands. A similar effect was realized by manipulating the electronic occupation of $3 \mathrm{~d}$ orbitals using electrical fields to control the magnetic anisotropy. ${ }^{31-35}$

\section{Conclusion}

We investigated magnetic properties of $\mathrm{Co}_{0.8} \mathrm{Fe}_{0.2}$ alloy nanomagnets formed on $\mathrm{Cu}$ (111) surface using spin-polarized STM. The alloy islands show a morphology, triangular shape and unfaulted/faulted stacking, similar to the Co islands formed on the same substrate. The spin-polarized tunneling spectra taken on $\mathrm{Co}_{0.8} \mathrm{Fe}_{0.2}$ islands show that both the majority and the minority states shift upward as compared to Co islands. For small islands (<7500 atoms), around $40 \%$ reduction in the coercivity is observed for $\mathrm{y}$ islands with respect to Co islands. By employing the Stoner-Wohlfarth model, the anisotropy constant of $\mathrm{Co}_{0.8} \mathrm{Fe}_{0.2}$ is found smaller by around $0.057 \mathrm{meV}$ per atom than that of Co. The observed anisotropy reduction is presumably due to the decreased valence electrons, which agrees qualitatively with the calcu- 
lation results by Daalderop et al. ${ }^{30}$ Using SP-STM, we demonstrate for the first time that by alloying Co islands with Fe, the coercivity of the individual nanomagnet is reduced.

\section{Conflicts of interest}

There are no conflicts to declare.

\section{Acknowledgements}

H.-H. Y. acknowledges the scholarship provided by the Alexander von Humboldt Foundation. This work is partially supported by Grants-in-Aid for Scientific Research from the Japan Society for the Promotion of Science (Grants No. JP16H02109, JP18K19013, and JP19H00859) and Ministry of Science and Technology of Taiwan under Grant No. MOST 1082112-M-003-011-MY2.

\section{References}

1 S. Bhanja, D. K. Karunaratne, R. Panchumarthy, S. Rajaram and S. Sarkar, Nat. Nanotechnol., 2016, 11, 177.

2 M. N. Leuenberger and D. Loss, Nature, 2001, 410, 798-793.

3 S. Ouazi, S. Wedekind, G. Rodary, H. Oka, D. Sander and J. Kirschner, Phys. Rev. Lett., 2012, 108, 107206.

4 O. Pietzsch, A. Kubetzka, M. Bode and R. Wiesendanger, Phys. Rev. Lett., 2004, 92, 057202.

5 H. Oka, P. A. Ignatiev, S. Wedekind, G. Rodary, L. Niebergall, V. S. Stepanyuk, D. Sander and J. Kirschner, Science, 2010, 327, 843-846.

6 M. Haze, Y. Yoshida and Y. Hasegawa, Phys. Rev. B: Condens. Matter Mater. Phys., 2017, 95, 060415(R).

7 S. Krause, L. Berbil-Bautista, G. Herzog, M. Bode and R. Wiesendanger, Science, 2007, 317, 1537-1540.

8 P. Mishra, Z. K. Qi, H. Oka, K. Nakamura and T. Komeda, Nano Lett., 2017, 17, 5843-5847.

9 K. Doi, E. Minamitani, S. Yamamoto, R. Arafune, Y. Yoshida, S. Watanabe and Y. Hasegawa, Phys. Rev. B: Condens. Matter Mater. Phys., 2015, 92, 064421.

10 G. Rodary, S. Wedekind, D. Sander and J. Kirschner, Jpn. J. Appl. Phys., 2008, 47, 9013-9015.

11 S. Wedekind, G. Rodary, J. Borme, S. Ouazi, Y. Nahas, M. Corbetta, H. Oka, D. Sander and J. Kirschner, IEEE Trans. Magn., 2011, 47, 3351-3354.

12 H. T. Jeng and D. S. Wang, J. Magn. Magn. Mater., 2007, 317, 46-52.

13 Y. Shiota, T. Maruyama, T. Nozaki, T. Shinjo, M. Shiraishi and Y. Suzuki, Appl. Phys. Express, 2009, 2, 063001.
14 A. Dittschar, M. Zharnikov, W. Kuch, M.-T. Lin, C. M. Schneider and J. Kirschner, Phys. Rev. B: Condens. Matter Mater. Phys., 1998, 57, R3209.

15 M.-T. Lin, W.-C. Lin, C.-C. Kuo and C.-L. Chiu, Phys. Rev. B: Condens. Matter Mater. Phys., 2000, 62, 14268-14272.

16 C.-C. Kuo, S.-F. Chuang, W. Pan, W.-C. Lin and M.-T. Lin, J. Appl. Phys., 2002, 91, 7185-7187.

17 P.-J. Hsu, C.-I. Lu, S.-W. Chen, W.-J. Hsueh, Y.-H. Chu, C.-H. Hsu, C. J. Butler and M.-T. Lin, Appl. Phys. Lett., 2010, 96, 142515.

18 G. Rodary, S. Wedekind, H. Oka, D. Sander and J. Kirschner, Appl. Phys. Lett., 2009, 95, 152513.

19 H.-H. Yang, C.-H. Hsu, W.-C. Lin and Y. Hasegawa, Phys. Rev. B: Condens. Matter Mater. Phys., 2021, 104, 035422.

20 W.-C. Lin, H.-Y. Chang, Y.-C. Hu and C.-C. Kuo, IEEE Trans. Magn., 2009, 45, 4037-4040.

21 I. Horcas, R. Fernández, J. M. Gómez-Rodríguez, J. Colchero, J. Gómez-Herrero and A. M. Baro, Rev. Sci. Instrum., 2007, 78, 13705.

22 A. L. Vázquez de Parga, F. J. García-Vidal and R. Miranda, Phys. Rev. Lett., 2000, 85, 4365.

23 M. V. Rastei, B. Heinrich, L. Limot, P. A. Ignatiev, V. S. Stepanyuk, P. Bruno and J. P. Bucher, Phys. Rev. Lett., 2007, 99, 246102.

24 L. Diekhöner, M. A. Schneider, A. N. Baranov, V. S. Stepanyuk, P. Bruno and K. Kern, Phys. Rev. Lett., 2003, 90, 236801.

25 S.-h. Phark, J. A. Fischer, M. Corbetta, D. Sander and J. Kirschner, Appl. Phys. Lett., 2013, 103, 032407.

26 A. Kubetzka, M. Bode, O. Pietzsch and R. Wiesendanger, Phys. Rev. Lett., 2002, 88, 057201.

27 R. C. O'Handley, Modern Magnetic Materials, Wiley, New York, 2000.

28 W. F. Brown JR., Phys. Rev., 1963, 130, 1677.

29 W. Wernsdorfer, Adv. Chem. Phy., 2001, 118, 99-190.

30 G. H. O. Daalderop, P. J. Kelly and M. F. H. Schuurmans, Phys. Rev. B: Condens. Matter Mater. Phys., 1990, 41, 11919.

31 C.-G. Duan, J. P. Velev, R. F. Sabirianov, Z. Zhu, J. Chu, S. S. Jaswal and E. Y. Tsymbal, Phys. Rev. Lett., 2008, 101, 137201.

32 M. Tsujikawa and T. Oda, Phys. Rev. Lett., 2009, 102, 247203.

33 K. Nakamura, R. Shimabukuro, Y. Fujiwara, T. Akiyama, T. Ito and A. J. Freeman, Phys. Rev. Lett., 2009, 102, 187201.

34 H. Zhang, M. Richter, K. Koepernik, I. Opahle, F. Tasnádi and H. Eschrig, New J. Phys., 2009, 11, 043007.

35 S. Ishibashi, T. Saruya, S. Murakami, T. Nozaki, S. Miwa, Y. Suzuki, T. Shinjo, K. Yakushiji, F. Bonell, Y. Shiota, S. Yuasa, H. Kubota and A. Fukushima, Nat. Phys., 2012, 8, 491-496. 